\title{
Le territoire comme lieu d'apprentissage et de construction de résilience sociale en Mésoamérique
}

\section{Arturo Curiel Ballesteros}

Traducteur : Lucie Sauvé et Marie-Ève Marleau

\section{OpenEdition}

\section{Journals}

Édition électronique

URL : http://journals.openedition.org/ere/4208

DOI : $10.4000 /$ ere.4208

ISSN : 2561-2271

Éditeur

Centr'ERE

Référence électronique

Arturo Curiel Ballesteros, «Le territoire comme lieu d'apprentissage et de construction de résilience sociale en Mésoamérique », Éducation relative à l'environnement [En ligne], Volume 5 | 2005, mis en ligne le 20 novembre 2005, consulté le 21 février 2020. URL : http://journals.openedition.org/ere/4208 ; DOI : 10.4000/ere.4208

Ce document a été généré automatiquement le 21 février 2020. 


\title{
Le territoire comme lieu
} d'apprentissage et de construction de résilience sociale en Mésoamérique

\author{
Arturo Curiel Ballesteros \\ Traduction : Lucie Sauvé et Marie-Ève Marleau
}

1 Le territoire où l'on vit est à la fois un creuset d'apprentissage et le reflet de ce que nous sommes. C'est un lieu de rencontre entre culture et nature, un espace de civilisation, mais aussi de chaos et d'incertitude. Or nous nous sommes approprié le territoire en le dominant, sans aucune logique de cohabitation et de communauté. Pour marquer notre territoire, nous avons choisi d'éliminer les manifestations de la vie et les valeurs que nous ne jugeons pas utiles.

2 On peut concevoir notre territoire environnemental comme un suprasystème où s'interpénètrent un système naturel et un système culturel. Ce dernier maintient l'ordre d'une civilisation qui est basée sur le pouvoir technologique et économique. Quant au système naturel, il peut être perçu comme une sorte de chaos: il se caractérise par sa dynamique mouvante empreinte d'incertitude, où se transforment sans cesse les diverses manifestations de la vie et où se tissent et se recomposent d'innombrables relations. Appréhendée selon une vision du monde positiviste, la valeur de la vie y est restreinte à celle de pourvoyeur de ressources naturelles destinées à répondre aux besoins du système culturel et à maintenir la structure de la civilisation dominante.

3 Pour contrer cette vision réductionniste, l'éducation relative à l'environnement invite à aborder le territoire, non pas seulement selon le paradigme positiviste qui tend à le modeler et à l'exploiter, mais aussi selon d'autres approches épistémologiques. Ainsi, Angel Maya (1997) met en lumière la dimension interprétative qui caractérise notre rapport au monde : 
Nous sommes seulement des interprètes du monde: notre lecture de ce qui se produit sur la Terre est celle de l'impact de notre culture. À travers l'éducation relative à l'environnement, nous transmettons le récit de ce que nous dit la Terre, de ce que nous disent les rivières, les montagnes dégradées, l'atmosphère irrespirable, la vie qui s'épuise [...]

Dans une approche positiviste, le territoire est « organisé » en fonction de la culture dominante et appréhendé selon une science objectiviste et dans des visées de transformation qui font appel au développement technologique. Le paradigme interprétatif considère le territoire comme un espace symbolique où s'exprime ou non, selon la culture de référence, la capacité de cohabitation avec la diversité des formes de vie et des cultures. Le paradigme de la critique sociale s'intéresse aux conditions de l'agir social qui font en sorte que le territoire devient un milieu communautaire, un patrimoine commun d'ordre naturel et culturel.

5 L'éducation, prise dans son sens le plus large et non limitée aux processus formels et institutionnels, doit stimuler la capacité d'articuler entre eux les savoirs issus des paradigmes positiviste, interprétatif et de la critique sociale. Il s'agit d'affiner la perception du milieu et de rendre plus pertinentes la définition des problèmes et l'identification des solutions relatives à l'aménagement d'un territoire caractérisé par la place qu'y prennent la nature et la possibilité de cohabitation des êtres. Le domaine de la gestion des risques offre un créneau de réflexion et d'intervention intéressant à cet égard.

\section{La résilience sociale et le territoire}

6 Toutes les formes de vie, lorsqu'elles interagissent sur un territoire, sont sujettes à des conditions de risques. Ces derniers peuvent être de différents ordres: ceux qui découlent des forces naturelles (comme les cycles géologiques, par exemple) et ceux qui résultent de la façon d'organiser une ville, de gérer une économie ou d'appliquer une technologie.

7 L'analyse d'un risque implique la reconnaissance de deux facteurs : l'existence d'une menace (ou condition déstabilisante) et la vulnérabilité ou le niveau de dommage que peut causer la menace en question. Il n'y a pas de risque tant que ne se manifeste pas la présence d'une menace; mais lorsque celle-ci apparaît sur un territoire, le niveau de dommage qu'elle peut occasionner dépend de la vulnérabilité du milieu, celle des systèmes écologiques comme celle du système social. À titre d'exemple de conditions de vulnérabilité d'une société, on peut mentionner les suivantes: le développement sans planification, la méconnaissance du milieu local, la dislocation sociale, la perte d'identité, le chômage et la pauvreté, la perte de valeurs, les difficultés de communication, les carences des services, la monoproduction ou monoculture et le manque de prévision.

8 Le concept de résilience fait référence à la capacité que possède un matériau, un système vivant ou un système social de se régénérer, de récupérer ses fonctions originelles après avoir été exposé à une perturbation externe. Initialement, le terme de résilience est apparu dans le domaine de la physique pour désigner la résistance de certains matériaux aux changements de conditions ou aux déformations. Ensuite, il s'est élargi au domaine de l'écologie pour désigner la capacité des écosystèmes à résister aux pressions anthropiques. En sciences humaines, ce concept fait maintenant 
référence à des personnes parvenant à traverser sans séquelles des expériences négatives, certaines pouvant même vivre au cœur de ces expériences un processus de développement personnel. Henderson et Miltein (2003), dans leur ouvrage portant sur la résilience à l'école, considèrent que l'éducation peut favoriser la construction d'une résilience sociale et aider à contrôler les facteurs de risque dans l'environnement à travers une démarche qui permet d'enrichir les liens sociaux, d'établir des limites claires et précises et d'enseigner des "habiletés pour la vie»; l'éducation peut construire la résilience par l'apport d'affection et d'accompagnement, par le maintien d'exigences élevées et par la mise en place de situations stimulant la participation à des projets signifiants.

9 Afin d'aborder le territoire où l'on vit, d'en faire un diagnostic, de déterminer les principes d'aménagement et d'identifier les conduites sociales appropriées, l'Institut National d'Écologie du Mexique (2000) propose une approche systémique et formule des questions clés. Par exemple :

Quels sont les facteurs culturels qui influencent l'état du système naturel au sein du territoire? Quelles menaces et quelles conditions de vulnérabilité peuvent affecter le patrimoine naturel et culturel du territoire? Quels éléments du système de production, du système social, écologique, culturel, politique et spirituel sont susceptibles de menacer la stabilité du territoire?

Les réponses à ces questions fournies par des experts scientifiques ou des agences gouvernementales résultent d'une approche analytique du territoire et peuvent avoir l'avantage d'intégrer une grande diversité d'éléments de problématiques et de solutions. Mais il est également important de considérer les différentes visions de la situation construites par chacun des divers groupes sociaux concernés.

\section{La participation : une condition de reconstruction de la résilience sociale}

11 Si l'on reconnaît que les représentations sociales se construisent entre autres à travers les expériences de vie qui affectent notre univers affectif, il devient certes essentiel de tenir compte de ces expériences pour mieux comprendre le territoire. Aborder la diversité des émotions liées à la construction des diverses représentations permet de mieux saisir un territoire, de concevoir des projets appropriés et de stimuler un agir social pertinent. À cet effet, il importe de considérer la relation au territoire des neuf groupes principaux identifiés dans l'Agenda 21 (ONU, 1993, chapitres 24 à 32), soit les femmes, les enfants et les jeunes, les autochtones, les organisations non gouvernementales, les autorités locales, les travailleurs et les syndicats, l'industrie et le commerce, les producteurs d'aliments et enfin, la communauté scientifique et technologique. Chacun de ces groupes est porteur d'expériences et de représentations particulières qu'il importe d'intégrer dans un processus de construction de la résilience d'un territoire.

Ces diverses visions et aspirations peuvent être en effet complémentaires ou confrontées dans la perspective d'un compromis, selon le contexte. Par exemple, les autorités locales, l'industrie et le commerce partagent en Méso-Amérique une vision commune du développement qui s'inscrit dans un paradigme positiviste. Pour ces groupes qui représentent l'idéologie dominante et le pouvoir de décision, le développement correspond à la subordination du système naturel à travers les 
infrastructures sociales et technologiques. Au contraire, les autochtones et les organisations non gouvernementales s'inscrivent le plus souvent dans un paradigme interprétatif ou de critique sociale: ils valorisent la nature et l'équité ; ils peuvent prendre la défense de la vie dans les milieux naturels et questionner le modèle de civilisation dominant.

Pour l'analyse des problématiques environnementales, dont celles qui impliquent certains niveaux de risque, il importe donc de stimuler la participation des principaux groupes qui détiennent le pouvoir (ceux qui sont associés à l'autorité locale, à l'industrie, au commerce, à la science et la technologie) tout comme celle des groupes qui sont exclus des processus de décision (tels les autochtones, les femmes et les jeunes). Les diverses expériences, perceptions et attentes relatives au système social et à la nature sont nécessaires à la compréhension globale des situations dans une perspective véritablement environnementale.

Il importe aussi de prendre conscience que pour l'identification des problèmes d'un territoire, notre éducation nous a entraînés à accorder une plus grande importance à ceux qui sont associés à des risques qui préoccupent davantage les groupes au pouvoir, en particulier ceux qui peuvent porter atteinte aux ressources. Ce qui diminue l'importance accordée aux problèmes liés à la vulnérabilité sociale, tels que perçus par les groupes qui ne détiennent pas le pouvoir. Par exemple, les travaux réalisés en MésoAmérique montrent que les problèmes identifiés comme étant les plus importants ont trait à la contamination de l'eau, aux déchets, à la déforestation et à l'accroissement accéléré de la population urbaine, qui sont en relation avec les menaces ou les pressions exercées sur le système naturel. Ce sont les problèmes que perçoivent davantage nos sens et qui nous affectent émotivement. C'est le cas par exemple, lorsque nous allons sur le bord d'une rivière ou d'un lac et que nous constatons la pollution de l'eau, nous sentons une odeur nauséabonde, nous prenons conscience que nous ne pouvons pas boire cette eau ni nous y baigner. De même, lorsque nous voyons les déchets qui encombrent les rues où nous vivons, les forêts clairsemées et les arbres rasés en milieu urbain, nous avons un sentiment de frustration, car dans ce type de territoire, ils constituent notre lien principal avec la nature. Nous sommes également affectés lorsque nous constatons que nos villes s'étalent jusqu'à envahir les flancs des montagnes, avec des conditions critiques de pauvreté depuis les quartiers du centre jusqu'en périphérie, où la situation s'aggrave encore davantage.

15 Cependant, bien que ces problèmes s'avèrent d'une extrême importance et qu'il est urgent d'intervenir, il ne suffit pas d'agir directement sur les effets observés. Les causes de ces problèmes liés à la dégradation des systèmes de vie se trouvent en amont, au cœur même des structures du système culturel. C'est ce dernier qui doit être fondamentalement reconstruit sans quoi les interventions de gestion environnementale resteront limitées. Par exemple, l'épuration de l'eau par différentes techniques, la collecte et l'enfouissement des déchets, la plantation massive d'arbres pour contrer la déforestation ou le fractionnement des villes en expansion pour en faciliter la gestion sont des stratégies qui répondent à la logique linéaire, positiviste et mécaniste des groupes qui détiennent le pouvoir, en laissant de côté les solutions que nous pourrions envisager si les problématiques étaient abordées selon une perspective interprétative ou de critique sociale.

En Méso-Amérique, comme dans plusieurs endroits de la planète, l'organisation du monde selon des critères économiques a occasionné au cours des deux cents dernières 
années de graves désordres dans la sociosphère, dans l'atmosphère, la lithosphère, l'hydrosphère et la biosphère; par ailleurs, la suprématie attribuée à la technologie à titre de solution à tous les problèmes de développement humain a conduit à l'échec.

[...] à la fin du vingtième siècle, nous avons découvert le côté obscur du chemin que nous avions commencé à tracer il y a huit cents ans, quand nous nous sommes séparés de la nature. Il est vrai que ce chemin nous a conduits au jaillissement de l'art, de la poésie, de la musique et de l'architecture de la Renaissance et au développement scientifique et technologique qui a suivi. Mais aussi, nous nous sommes approprié le terrain vague du progrès et du développement illimités qui s'inscrivent maintenant dans le paradigme mécaniste [...]. Or l'image de notre planète bleue vue de l'espace est devenue une icône nous rappelant la nécessité d'adopter une nouvelle perspective holistique [...]. Si, sur la photographie de la Terre, grâce au zoom d'un satellite, nous dirigeons notre regard sur les forêts tropicales d'Amazonie, nous pouvons observer que des grandes zones continuent d'être détruites. Pourquoi cela doit-il nous préoccuper? La réponse mécaniste est que même si les choses nous échappent pour l'instant, nous serons toujours capables de contrôler la situation par la reforestation et la gestion des forêts. Cependant la théorie du chaos nous apprend que nos interventions ont leurs limites et que les résultats sont toujours, jusqu'à un certain point, imprévisibles. Supposer que les nouvelles générations seront capables de développer la technologie nécessaire pour pallier les dommages que nous sommes en train de causer maintenant, correspond à une dangereuse illusion [...] (Briggs et Peat, 1999, p. 203, 208-210)

17 Pour l'analyse de problèmes qui ont une «motricité » majeure, c'est-à-dire dont la résolution est susceptible d'influencer favorablement celle d'autres problèmes relatifs à la structure du système culturel, la participation des principaux groupes sociaux d'un territoire devient nécessaire. En effet, leurs différentes perceptions du problème relèvent à la fois du domaine affectif et du domaine intellectuel et sont de nature à mieux éclairer la situation. Or, en Méso-Amérique, les problèmes « moteurs » qui ont été identifiés sont le manque de participation sociale équitable, représentative et informée, le manque de coordination institutionnelle, le manque de planification et la perte d'identité et de valeurs. Ces problèmes sont étroitement liés à la vulnérabilité du territoire. Une société qui ne participe pas, qui ne se coordonne pas, qui ne planifie pas et qui perd son identité et ses valeurs, est une société vulnérable à l'égard des conditions de risques.

18 Sans participation des communautés, l'économie restera dominante dans l'aménagement du territoire, augmentant ainsi les coûts sociaux et écologiques d'un tel choix de gouvernance. Sans coordination institutionnelle, l'aménagement du territoire restera inadéquat, affectant les systèmes vitaux et diminuant la capacité de gestion intégrée. Sans concertation pour un travail collectif et sans planification, on ne peut mettre en œuvre un plan d'aménagement: on ne peut pas contrer par exemple, le gaspillage d'énergie, le mauvais usage du territoire et la perte d'identité. Or, sans identité (liée à l'estime de soi, au respect de soi-même, de sa culture, de son milieu de vie), nous n'aurons pas de patrimoine. Au bout du compte, la pollution des rivières, l'accumulation des déchets et la déforestation sont reliées à la perte d'identité et de valeurs (perte de pouvoir et de vouloir), à laquelle s'ajoutent la corruption et la transgression de la législation, particulièrement en Méso-Amérique. Pourtant, celle-ci a jadis été un territoire où l'identité des peuples s'appuyait sur une certaine vision de la relation entre la culture et la nature. Un exemple nous en est donné par Roberto A. 
Restrepo (2000) dans son œuvre El rostro de la serpiente, où il met en évidence le rôle de l'eau comme culture et miroir des civilisations :

En Méso-Amérique, la gestion de l'eau, de la terre et de la vie elle-même était caractérisée par un dialogue équitable, marqué d'un profond respect. Aujourd'hui, avec la désacralisation du monde, l'eau, la terre et la vie peuvent être mesurées en simples termes économiques. Un tel mauvais traitement ne cesse de se répéter et devient un problème de survie au cours du 21e siècle. Les lieux qui étaient considérés jusqu'ici comme des sanctuaires, où l'on s'alimentait de foi et d'espérance et où l'on rendait un culte à l'eau, à la terre et à la vie, se remplissent des saletés que nous produisons en tant qu'êtres civilisés, industrialisés et pratiques. Ainsi, les visions cosmologiques millénaires disparaissent tout comme l'eau et la vie, pendant que notre civilisation réinvente des systèmes sans toutefois transformer nos consciences.

La participation des communautés enrichit les liens sociaux et contribue à construire la signification de notre relation au territoire. La planification collective de l'aménagement et de la gestion de ce dernier permet de fixer des objectifs et d'établir des limites claires et précises. Enfin, la consolidation d'une identité sociale et l'affirmation de valeurs culturelles sont essentielles pour construire une relation affective au milieu de vie et stimuler le désir de le préserver ou de l'améliorer. Tout cela contribue à la résilience du territoire.

\section{Thématiques pour une éducation à la résilience territoriale}

L'éducation relative à l'environnement peut contribuer à la transformation de la logique utilitariste, individualiste et à courte vue des gens qui habitent et partagent un territoire. Elle peut stimuler la participation et aider à modifier les scénarios qui conduisent à l'accroissement des risques dans un territoire par l'augmentation de la vulnérabilité de ce dernier. Elle devient alors une éducation à la résilience territoriale.

21 À cet effet, l'éducation relative à l'environnement adopte les caractéristiques suivantes :

1. elle est centrée sur la relation des personnes au territoire local, tenant compte des relations avec l'environnement global ;

2. elle se penche sur les risques territoriaux;

3. elle tente de contribuer à la diminution de la vulnérabilité individuelle et communautaire ;

4. elle intègre entre elles les dimensions écologique, politique, économique et culturelle des réalités territoriales ;

5. elle stimule l'action sociale et la participation communautaire ;

6. elle encourage la solidarité, l'engagement et la responsabilité sociale ;

7. elle favorise la coordination entre les institutions ;

8. elle incite à la planification à court, à moyen et à long terme ;

9. elle développe non seulement des habiletés, mais aussi des attitudes ;

10. elle incite à la clarification et à l'affirmation des valeurs ;

11. elle reconnaît l'importance de l'émotivité comme mode de relation au territoire: elle stimule entre autres l'affection et l'entraide. 
Les thématiques suivantes sont particulièrement intéressantes pour inspirer des projets ou des programmes d'éducation relative à l'environnement qui intègrent ces onze caractéristiques :

\section{La nourriture}

23 La nourriture unit les personnes au territoire. Le maïs, le cacao, la vanille, les mets à base de tomates sont parmi les aliments caractéristiques de la Méso-Amérique, qui contribuent par ailleurs à l'apport alimentaire mondial. Dans cette région, on observe aussi une riche culture culinaire où le rôle de la femme a été crucial pour la conservation des espèces alimentaires locales. Pour aborder cette thématique de la nourriture, il importe d'intégrer les divers aspects des réalités associées, soit les aspects écologiques (sol, pluie, semences, etc.), politiques (dont la sécurité des aliments et l'utilisation équitable des terres), économiques (comme la question des subsides ou celle de la commercialisation des produits) et sociaux (entre autres, la culture culinaire). Mais plus encore, la nourriture fait partie de l'identité et des valeurs du territoire mésoaméricain; aborder ensemble le thème de la nourriture amène à considérer et à reconstruire les liens sociaux. Cela implique le développement d'attitudes relatives à l'autonomie (comme celle de préférer les produits locaux) et de compétences relatives à la diversification des productions vivrières, de façon à réduire la dépendance à l'égard des produits exogènes. Cela peut aussi contribuer à faire naître ou renforcer une solidarité avec les communautés rurales productrices d'aliments, leur donnant ainsi affection et appui. Il importe en particulier d'offrir aux producteurs et aux femmes des occasions de participation signifiantes et de stimuler l'engagement à l'égard de la protection des zones productives du territoire. Une éducation relative à l'environnement centrée sur le thème de la nourriture peut ainsi contribuer à la diminution de la vulnérabilité sociale à l'égard des risques.

\section{La santé environnementale}

La santé environnementale met en évidence la relation étroite entre les communautés humaines et les éléments naturels de leur territoire, comme ceux qui sont liés au cycle hydrologique. Aborder ce thème amène à clarifier les liens entre les dimensions écologique (l'eau, l'air, le sol, etc.), politique (les lois, les normes, les règlements, la planification liée à la sécurité), économique (la santé et la sécurité au travail) et sociale (la santé publique) des réalités concernées. La santé environnementale fait également appel à la participation communautaire et à l'action sociale pour la réduction des risques au sein des milieux de vie, de travail et de loisirs. C'est un enjeu public de nature à favoriser la solidarité entre les individus du présent et du futur, à engendrer le sens de l'engagement et à stimuler la responsabilité sociale. La santé environnementale interpelle en effet la participation des gouvernements locaux, de la communauté scientifique, du secteur de l'industrie, des syndicats, des organisations non gouvernementales et des autochtones. En santé environnementale, il s'agit d'identifier les menaces (comme les diverses formes de pollution) et les conditions de vulnérabilité (par exemple, celles qui sont liées aux caractéristiques démographiques des bassins de populations qui sont exposés aux risques); il s'agit également de stimuler la coordination institutionnelle et la planification, l'identification de limites et la multiplication des occasions de participation communautaire. 


\section{La diversité environnementale}

25 dimensions naturelles et culturelles de leur territoire. Aborder cette problématique implique la prise en compte de ses dimensions écologique (biodiversité, diversité des espèces et des habitats, des paysages), politique (politique de protection du patrimoine, zones naturelles protégées), économique (diversification des productions) et sociale (diversité culturelle, sociodiversité). Également, la conservation de la diversité biologique, culturelle et " productive » doit être prise en charge par une action sociale qui favorise la solidarité entre les populations, de même que la promotion et la valorisation des diverses cultures et courants de pensée. Cela fait appel à la responsabilité sociale et à la coordination des groupes en présence, soit les organismes gouvernementaux et non gouvernementaux, les entrepreneurs, les autochtones et autres. Promouvoir la diversité implique le développement d'habiletés d'interprétation et de gestion environnementale, et aussi des attitudes comme la participation active, le travail d'équipe et l'ouverture à d'autres façons de penser et de vivre. La diversité fait référence à des valeurs, tels la tolérance, la sensibilité esthétique, l'engagement, l'amour de la nature, l'égalité, le respect, le dialogue et la paix. La perte de diversité des formes de vie, de pensée et de savoirs, tout comme la perte des valeurs liées à la nature et à la culture, augmentent la vulnérabilité socioécologique. Au contraire, la diversité est de nature à enrichir les liens sociaux. Travailler à sa promotion implique le développement d'" habiletés pour la vie ", stimule l'affection et l'entraide et met en place des occasions de participation communautaire à des projets signifiants.

\section{Le risque technologique et socio-organisationnel}

La gestion du risque implique l'engagement d'une communauté dans l'identification et l'investigation des menaces à l'égard de son territoire et dans l'évaluation de la vulnérabilité de ce dernier; elle fait appel à la participation communautaire et à l'action sociale pour contrer la menace et réduire une telle vulnérabilité. Une éducation aux risques peut accroître la solidarité avec les communautés vulnérables et au sein de ces dernières. Elle invite à la recherche d'informations et de solutions, et conduit au choix responsable de stratégies de communication appropriées. À cet effet, il importe de tenir compte de la complexité des liens entre les dimensions écologique (accès aux ressources naturelles de qualité), politique (le concept de "gouvernance»), économique (coûts générés par la pollution) et sociale (capacité de réponse) des situations concernées. Aborder le risque requiert également le développement d'habiletés relatives à l'analyse systémique, à l'interdisciplinarité, à la communication, à la gestion, à l'évaluation, etc.; cela suppose aussi le développement d'attitudes comme la coopération, la prévention et le travail d'équipe. La gestion du risque implique la coordination entre les scientifiques, les industriels, les instances gouvernementales, les organisations non gouvernementales et autres groupes sociaux. Elle requiert une planification à court terme pour réduire la vulnérabilité et à long terme pour diminuer et prévenir les menaces : il s'agit d'atteindre un niveau de risque acceptable, en accord avec la valeur de la vie. Prendre en charge collectivement une situation de risque est de nature à enrichir les liens sociaux ; cela suppose de convenir ensemble de limites claires et précises et de stimuler la participation communautaire. 


\section{La gestion de l'eau}

\section{effet une nécessité de première importance et un enjeu social majeur, tant à l'échelle} locale que globale : l'eau fait partie de l'agenda environnemental de tous les pays et elle devient également, de plus en plus, l'objet de litiges régionaux et internationaux. La gestion de l'eau est un acte social : elle fait appel à la participation communautaire et à l'engagement responsable des divers acteurs, décideurs et autres, qui doivent coordonner leurs efforts entre eux. Elle interpelle la solidarité avec les différentes formes de vie sur le territoire, entre les divers groupes culturels et avec les générations futures. La gestion de l'eau implique la prise en compte de ses dimensions écologique (cycle hydrologique), politique (l'accès à l'eau sur le territoire), économique (l'eau dans les activités de production) et sociale (l'eau et la santé, l'eau en tant qu'élément dominant de la culture). L'identification des diverses menaces au sein d'un territoire amène à clarifier leurs liens avec l'excès, la carence et la contamination de l'eau. L'examen du niveau de vulnérabilité d'une communauté permet de constater que celleci est souvent principalement reliée à la perte de l'accès à cette ressource vitale. Comme pour les thématiques précédentes, aborder collectivement la gestion de l'eau est de nature à enrichir les liens sociaux, à développer des " habiletés pour la vie ", à renforcer l'affection et l'entraide nécessaires à la résolution des problèmes et à mettre en place des occasions signifiantes de participation communautaire.

Les thématiques que nous venons d'esquisser s'avèrent porteuses pour la construction ou reconstruction de la résilience au sein des populations concernées. Elles peuvent inspirer une éducation relative à l'environnement axée sur le développement de sociétés plus équitables, plus respectueuses des diverses formes de vie. Elles peuvent être abordées selon les approches positiviste, interprétative et de la critique sociale, dont les savoirs combinés offrent une meilleure compréhension du territoire, permettant ainsi d'ouvrir des perspectives de changement plus appropriées.

\section{BIBLIOGRAPHIE}

Angel Maya, A. (1997). Discurso de Augusto Angel Maya al otorgarle la Universidad de Guadalajara el Doctorado Honoris Causa. Vidéo. Guadalajara : Rectorat de la Universidad de Guadalajara.

Briggs, J. et Peat, F.D. (1999). Las Siete Leyes del Caos - Las Ventajas de una Vida Caótica. Barcelone : Grijalbo.

Henderson, N.Y. et Miltein, M.M. (2003). Resiliencia en la Escuela. Buenos Aires : Piados. Instituto Nacional de Ecología. (2000). Ordenamiento Ecológico General del Territorio. Memoria Técnica 1995-2000. Cédérom. México : INE, SEMARNAP. Organisation des Nations Unies - ONU. (1993). Agenda XXI. México : SEDESOL.

Restrepo, R.A. (2000). El rostro de la serpiente ; el manejo prehispánico del agua. Cédérom. Paris : UNESCO. 


\section{AUTEURS}

\section{ARTURO CURIEL BALLESTEROS}

Détenant un doctorat en sciences biologiques de la Universidad Autónoma de Madrid, il est chercheur en éducation relative à l'environnement et à la santé environnementale à l'Institut de l'environnement et des communautés humaines de la Universidad de Guadalajara, au Mexique. 